\title{
Auroral Cluster: A Space Physics Mission for Multiple, Electronically Tethered Small Satellites
}

\author{
J.P. Andrews, J.R. Scherrer, J.H. Waite, and J.L. Burch \\ Southwest Research Institute \\ Instrumentation and Space Research Division
}

\begin{abstract}
Auroral Cluster is a space physics mission that has been identified by the NASA Space Physics Strategic Implementation Study as a candidate for flight in the next decade. Auroral Cluster will employ multiple spacecraft outfitted with similar complements of science instruments allowing simultaneous multipoint plasma measurements in the Earth's auroral regions. Co-orbiting small satellites (mass < $400 \mathrm{~kg}$ each) that are electronically "tethered" to share distributed spacecraft systems represent an efficient approach for achieving the science goals of the Auroral Cluster mission. Multisatellite missions represent a new trend in gathering space science data and pose many new and difficult challenges for the space systems engineer. The results of an Auroral Cluster feasibility study, which discusses a variety of mission trade-offs, are presented. A discussion of the science background and mission goals is used to identify the technical drivers for the design of the multiple spacecraft system. A mission plan and some considerations for a Auroral Cluster satellite design are presented. Special consideration is given to the spacecraft subsystems that will allow the system to be operated as a network of electronically tethered interdependent small satellites. These subsystems include attitude determination, spatial separation knowledge and control, data storage, and intersatellite communication.
\end{abstract}

\section{INTRODUCTION}

The Earth's magnetosphere is the site of many complex plasma phenomena which play central roles in solar-terrestrial physics and upper atmosphere geophysics and which have counterparts in other planetary and astrophysical environments. The aurora is one of the most dynamic phenomena in the magnetosphere and provides a visible manifestation of the final state of the energy transfer that results from the interaction of the solar wind with the magnetosphere. It is both interesting and important to our concepts of how magnetospheres work, and we do not fully understand it. Auroras are easily accessible to both sounding rockets and orbiting spacecraft, and the important parameters, which include suprathermal particle distributions, electric fields and magnetic fields, are measurable with high accuracy. Therefore, there must be some unresolved problem with our measurement techniques, which has hindered our ability to fully understand the physics of the auroral regions. This problem is driven by the fact that the aurora is formed by three-dimensional, time-varying phenomena that must be diagnosed with a three-dimensional array of spacecraft with high data rates. Sounding rockets cannot reach the altitudes where the auroral particle acceleration region is known to be $\left(1\right.$ to $\left.2 R_{E}\right)$, and auroral-physics spacecraft have been deployed singly and with low data rates. ${ }^{1}$

To overcome the problems associated with using one spacecraft to resolve the dynamic phenomena occurring in the magnetosphere, a variety of multiprobe missions have been proposed which would allow independent spatial and temporal measurements of the plasma phenomena to be made. Several of these candidate multiprobe, or cluster, missions are proposed as future magnetospheric physics missions and are discussed in NASA's Space Physics Strategy Implementation Study. ${ }^{2}$ Among the several cluster programs identified in the study (each of which is designed to examine different regions of the magnetosphere), the Auroral Cluster mission presents a unique opportunity to apply small satellite technology and capabilities. 
Although auroral phenomena are very prominent at low altitudes, we now know that the precipitating electrons are accelerated at higher altitudes of one $\mathrm{R}_{\mathrm{E}}$ or so. Cluster-type measurements are crucial for our understanding of these acceleration regions. While not universally accepted, the concept of an auroral potential structure, involving electric-field components both perpendicular and parallel to the magnetic direction, is used extensively in experimental and theoretical studies of the aurora. The spatial dependence of the auroral potential structures, their temporal variations, and the relationship between the resulting large-scale inverted- $V$ electron precipitation patterns and the generally smaller-scale auroral arc structures are topics of fundamental importance to our eventual understanding of auroral particle acceleration and magnetosphere-ionosphere coupling.

The prime objective of the Auroral Cluster mission will be to resolve temporally and spatially varying plasma phenomena in the auroral regions with a resolution that varies over the range of -.1 to 100 kilometers. Some specific phenomena of interest include auroral electron acceleration, transverse and parallel ion acceleration, cusp plasma entry and transport, Birkeland current systems, ionospheric plasma outflow, the dynamics of ionospheric convection patterns, and the generation of auroral plasma waves. These phenomena all play an important role in the exchange of momentum and energy between the magnetospheric and ionospheric elements of the Earth system. By using four independent instrument platforms, it is possible to obtain multipoint measurements of $\mathbf{E}$ and $\mathbf{B}$ and then derive the vector properties of the auroral regions. Specifically, the measurements would entail deriving the curls, gradients, and time derivatives of $\mathbf{B}$ and $\mathbf{E}$, as well as the convective derivatives of the bulk plasma parameters. To accomplish these goals the mission's instrument complement must be capable of measuring electric fields (DC-1Mhz), magnetic fields (DC-10kHz), 3-D electron distributions $(-10 \mathrm{eV}-30 \mathrm{keV}), 3-\mathrm{D}$ ions with composition $(-10 \mathrm{eV}-30 \mathrm{KeV})$, and wave-particle correlations.

\section{MISSION REQUIREMENTS}

The Auroral Cluster (AC) mission requirements are determined by a unique set of scientific objectives and present novel engineering challenges that must be overcome in order to implement the program. A summary of the primary $\mathrm{AC}$ mission requirements is provided in Table I found at the end of this section. Foremost amongst these requirements is the need to use interdependent multiple science platforms capable of providing simultaneous multipoint plasma measurements. It is obvious that the multiplatform requirement will have a tremendous impact on the design and operation of all segments of the AC mission. Further complicating the mission requirements is the need to fly the platforms in a configuration that allows for adequate and variable interplatform separation and positioning while the cluster traverses the spatial regions of scientific interest. In addition, it is mandatory that the attitude, position, and operational sequencing of each science platform be known very precisely in order to generate the high-resolution plasma and field quantities, i.e., $\nabla \times B, \partial \mathrm{n} / \partial \mathrm{t}+\mathrm{v} \bullet \nabla \mathrm{n}$, etc. Main science objectives of the Auroral Cluster mission which determine these mission requirements include (1) the determination of the current density and vorticity through measurements from the cluster configuration, (2) resolution of the ambiguity between temporal and spatial variations of plasmas and fields, and (3) investigation of the generation and propagation of plasma waves and their interaction with the plasma.

\section{Current Density and Vorticity}

Field-aligned currents play a central role in the transfer of electrical energy between the solar wind and the Earth's magnetosphere, ionosphere, and atmosphere. By measuring the perturbations in the 
geomagnetic field produced by these currents, it has been determined that they are a permanent feature of the auroral oval and that they tend to occur in upward-downward pairs in a longitudinally-extended current-sheet configuration. Any measurement of the actual current density using a single spacecraft has of necessity required assumptions concerning the spatial configuration of the current systems. Such assumptions break down completely in highly structured regions such as auroral arcs. This difficulty can be overcome by making simultaneous measurements at several locations within a volume over which the current density is assumed to be relatively uniform. A minimum of four such measurements is required to determine the spatial gradients of the three magnetic field components, which can then be used to calculate $\nabla \times \mathbf{B}$ and the current using Ampere's Law, $\nabla \times \mathbf{B}=\mu_{0} \mathrm{~J}$; where the displacement currents have been neglected as a result of the macroscopic scale of the currents being measured. The optimum configuration for using four spacecraft to obtain measurements of three spatial gradients is one that places a spacecraft at each of the four comers of a tetrahedron, as shown in figure 1.

Measurement of field-aligned currents is certainly one of the most powerful uses of closely-spaced spacecraft arrays. However, it places stringent requirements on measurement accuracy and on spacecraft position and attitude knowledge, upon which measurement accuracy directly depends. To illustrate this it is necessary to express Ampere's law in the form:

$$
\mathbf{J}=(\nabla \times \mathbf{B}) / \mu_{0} \approx \Delta \mathbf{B} /\left(\mu_{0} \mathrm{~d}\right)
$$

where $d$ is the spatial separation over which the magnetic field perturbation is observed. The ability to detect a distributed current density by direct measurement of the curl of $\mathbf{B}$ among multiple spacecraft in a background magnetic field $\mathbf{B}_{0}$ is governed by the accuracy of the spacecraft attitude determination, $\alpha$ (measured in degrees), and the ability to determine the inter-spacecraft range, and can

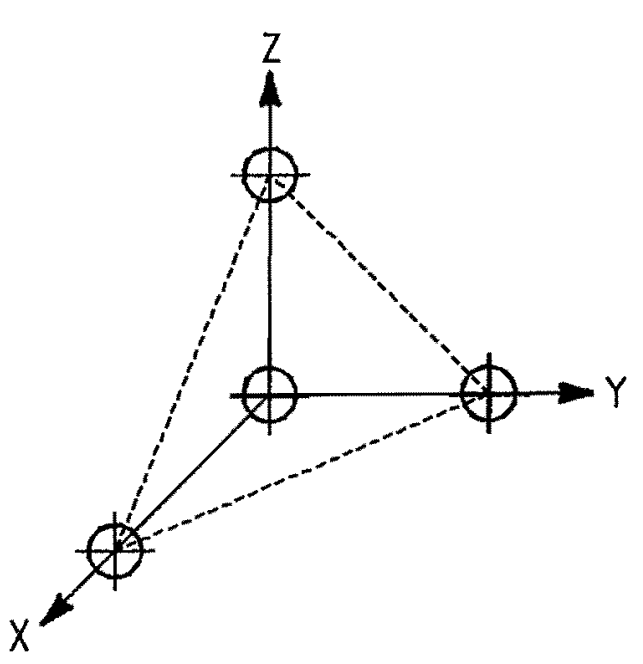
be stated as

$$
\Delta \mathrm{B} / \mathrm{B}_{0}>\left(2 \pi / 360^{\circ}\right)(\alpha)
$$

Combining the above two formulas, the minimum current density detectable is

$$
J_{\min }\left(n A / m^{2}\right) \approx 14 B_{0} \alpha / d(n T \operatorname{deg} / k m)
$$

Using values of $7000 \mathrm{nT}$ for $\mathrm{B}_{0}, \mathrm{~d}=10 \mathrm{~km}$, and $\alpha=.01$ gives a minimum current density of $100 \mathrm{nA} / \mathrm{m}^{2}$, which is appropriate to the auroral zone and implies that an attitude knowledge of at least .01 degrees is required for each instrument platform. 


\section{Spatial/Temporal Variations}

Due to the quantitative nature of the Auroral Cluster mission, the observations will deal directly with the underlying physics and hence moments of the kinetic equation for a distribution function f:

$$
\frac{\partial f}{\partial t}+v \cdot \frac{\partial f}{\partial r}+\frac{F}{m} \cdot \frac{\partial f}{\partial v}=\left(\frac{\partial f}{\partial t}\right)_{e}
$$

where the collision term on the right hand side may be due to wave-particle interactions. Maxwell's equations with their spatial variation may also be used to examine mesoscale and microscale processes directly.

$$
\begin{gathered}
\nabla \times \mathbf{E}(\boldsymbol{r}, t)=-\frac{\partial \boldsymbol{B}(\boldsymbol{r}, t)}{\partial t} \\
\left.\nabla \times \mathbf{B}(\boldsymbol{r}, t)=\mu_{0} \boldsymbol{J}+\mu_{0} \varepsilon_{0}\right) \frac{\partial \boldsymbol{E}}{\partial t} \\
\nabla \cdot \mathbf{E}(\boldsymbol{r}, t)=0 \text { (quasineutrality) } \\
\nabla \cdot \mathbf{B}(\boldsymbol{r}, t)=0
\end{gathered}
$$

In the case of electric and magnetic field variations the kinetic equation may be used directly with Maxwell's equation and the effects of spatial inhomogeneities may be considered.

Separation of space and time variation in these plasma and field quantities requires measuring transported quantities such as electron and ion density in the convecting plasma frame, i.e. the use of the convective derivative $\partial \mathrm{n} / \partial \mathrm{t}+\mathrm{v} \cdot \nabla \mathrm{n}$. Assuming that the velocity is on the order of the spacecraft velocity, $-5 \mathrm{~km} / \mathrm{s}$, and that the minimum separation distance of the spacecraft is $\mathrm{d} \sim 10 \mathrm{~km}$, measurement of the ion convective derivative requires a time resolution $\Delta t$ of the order

$$
\frac{1}{\Delta \mathrm{t}}-\frac{\mathrm{v}}{\mathrm{d}}=\frac{5}{10}=0.5 ; \Delta \mathrm{t}-2
$$

which is accomplished by a spacecraft spin rate of $20 \mathrm{rpm}$ and a 2-dimensional ion detector mounted with its axis of symetry perpendicular to the spacecraft spin axis. Higher convection velocities require larger separation distances of the spacecraft to maintain proper temporal resolution with respect to the ion distribution function. However, in the case of electrons, where higher temporal resolution for measuring the distribution function may be available $(-10 \mathrm{~ms})$, the use of multipoint measurements by a single satellite may be desirable to resolve properly the terms from equation (9):

$$
\Delta \mathrm{t} \approx 0.01=\mathrm{d}^{\prime} / \mathrm{v} \Rightarrow \mathrm{d}^{\prime}-50 \mathrm{~m}
$$


where $d^{\prime}$ is the separation distance between antenna sensors on a single spacecraft.

\section{Plasma Waves}

The generation of plasma waves is of fundamental importance in dissipating and redistributing the free energy of auroral processes. These waves appear to play a role both in ion and electron acceleration processes and in the electromagnetic transport of energy into and out of the auroral system. Several possible examples (and the mission requirements that they suggest) are discussed below.

Electron acceleration is a key factor in auroral processes. Many suggestions for how this acceleration takes place have been proposed. One such example is double layers, which can be detected by their effects on the electric fields and electron distributions. The detection of these structures requires that simultaneous electron and wave measurements be carried out at frequencies near the plasma frequency (3-10 kHz in the high-altitude auroral region). This implies pitch angle measurements of electrons with time scales of the order of $1 \mathrm{~ms}$ and scale lengths for electric field measurements on the order of $100 \mathrm{~m}$. Propagation of these structures along the magnetic field is expected to occur at the ion sound speed, which is of the order of $10 \mathrm{~km} / \mathrm{s}$. This implies an interspacecraft delay of $\sim 1 \mathrm{~s}$ for a $10 \mathrm{~km}$ separation distance.

Although the use of interferometric methods for the various wave antenna on a given spacecraft is adequate for determining the wave propagation vector for plasma waves with wavelengths of the order of the antenna lengths (100-200 m), other waves such as the Auroral Kilometric Radiation and Alfvén waves have much larger wavelengths (AKR $50-600 \mathrm{kHz}$; wavelengths $-6,000$ to $500 \mathrm{~m}$ ). In the case of $A K R$, the wave propagation vector can be studied if the separate spacecraft clocks are synchronized to provide timing information on the order of $100 \mathrm{~ns}$. Away from the auroral source region, the interferometric wave detection can give information about the characteristics of the source location and time variability. For Alfven waves, the wavelengths are much larger $(\sim 1000 \mathrm{~km})$ and their frequencies lower $(100 \mathrm{~Hz})$; however, their propagation along the magnetic field can be studied by interspacecraft correlation of the observed waves. Interspacecraft separations of $10 \mathrm{~km}$ imply again an interspacecraft temporal resolution of 100 ns to study Alfven propagation.

Ion cyclotron waves have spatial scales of the order of the ion gyroradius $\left(10 \mathrm{~km}\right.$ for a $\mathrm{keV} \mathrm{\textrm {O } ^ { + }}$ ion) and frequencies of $\sim 10 \mathrm{~Hz}$ for $\mathrm{O}^{+}\left(100 \mathrm{~Hz}\right.$ for $\left.\mathrm{H}^{+}\right)$. The spatial structure and wave-particle interactions can be studied by interspacecraft correlations of the plasma wave and ion detector data if the temporal resolution of the measurements is on the order of 1-10 milliseconds.

In summary, plasma waves require synchronized sampling/timing on the order of $100 \mathrm{~ns}$ and interspacecraft correlators if fundamental questions about the microphysics of auroral processes are to be addressed.

\section{MISSION DESIGN}

The goal of the mission design process is to formulate a system architecture that can meet or exceed the stated mission requirements at a minimal cost. It is both possible and desirable to achieve the AC mission objective by employing four, small, "electronically tethered" satellites. Each of the satellites would be fully equipped to serve as a science instrument platform from which the desired simultaneous multipoint auroral plasma measurements would be made. The keys to employing a cost effective small satellite architecture, however, will be the judicious selection of a science payload capable of obtaining 
Table I Auroral Cluster Primary Mission Requirements Summary

$\begin{array}{ll}\begin{array}{l}\text { PARAMETER/CONSTRAINT/REQUIREMENT/DESIGN SPECIFICATION } \\ \text { General Configuration }\end{array} & \begin{array}{l}4 \text { "electronically tethered" spacecraft } \\ \text { Orbit/Dynamics }\end{array} \\ \begin{array}{l}\text { High-altitude, high-inclination orbit maximizing time spent in } \\ \text { auroral regions }\end{array} \\ \begin{array}{l}1 \text { year design life } \\ \text { Launch Vehicle }\end{array} \\ \begin{array}{l}\text { Desire single launch for all } 4 \text { satellites } \\ \text { Communications }\end{array} \\ \begin{array}{l}\text { System must provide the intersatellite electronic tether and the } \\ \text { ground link }\end{array} \\ \text { Attitude } & \begin{array}{l}\text { Knowledge to .01 } \\ \text { Intersatellite position knowledge to } 1 \% \text { or } 100 \mathrm{~m}\end{array} \\ \text { Position } & \begin{array}{l}\text { Minimize charge build-up. All surfaces exposed to plasma to } \\ \text { be conductive }\end{array} \\ \text { Electrostatic Cleanliness } & \text { Multiple data taking modes }\end{array}$

the desired measurements and the limited use of advanced spacecraft subsystem technology. To demonstrate the feasibility of using small satellites for the AC mission, the following sections will discuss the orbital options considered for the mission, a strawman science payload for the mission, and the possible configurations of the AC spacecraft's subsystems.

\section{Orbital Considerations}

The ideal orbit for the $\mathrm{AC}$ mission should maximize the frequency at which the cluster traverses the Earth's auroral acceleration regions, while simultaneously allowing for the minimal cluster velocity when passing through those regions. There are also a variety of possible mission-driven constraints on the longitudes of the orbit's nodes, which would control the relative times that the cluster traverses the auroral regions. In addition, the various engineering issues, i.e., thermal management, power generation, RF link considerations, etc., must be factored into the orbital design process. Finally, the optimum orbit will maximize the mission's science return while minimizing the launch vehicle requirements. Three likely orbital candidates were arrived at after these constraints and issues were considered. These candidate orbits are (1) high-apogee, high-perigee, polar (HAHPP) orbit; (2) high-apogee sun-synchronous (HASS) orbit; and (3) sun-synchronous, fixed line of apsides (SSFLA) orbit. The pertinent orbital parameters for these orbits are listed in Table II and a brief discussion of them follows.

\section{HAHPP Orbit}

The high-apogee $(-10,000 \mathrm{~km})$, high-perigee $(-5,000 \mathrm{~km})$, polar $\left(\mathrm{i}=90^{\circ}\right)$ orbit is ideal for maximizing the time the cluster spends in the auroral acceleration regions because it provides a high- 
altitude $(>5,000 \mathrm{~km})$ traversal of both the north and south auroral regions during each orbit. Unfortunately, this orbit is obtainable only with considerable $\Delta V$ cost and, therefore, is the most payloadmass-restrictive orbital candidate. The cluster's velocity in this orbit will be $6.43 \mathrm{~km} / \mathrm{s}$ at perigee and 4.47 $\mathrm{km} / \mathrm{s}$ at apogee. If the orbit is held at a $90^{\circ}$ inclination there will be no nodal precession; this will influence the satellites' thermal management and power generation subsystems design because of the variable position of the solar flux vector over the mission. Since the orbit plane is fixed in inertial space, measurements of the auroral spatial regions can be made over all local times during the course of a oneyear mission. The orbit's line of apsides rotates at $-.35^{\circ}$ per day, allowing the cluster to traverse the auroral regions at altitudes that vary from $5,000 \mathrm{~km}$ to $10,000 \mathrm{~km}$ over the course of a year.

\section{HASS Orbit}

The high-apogee sun-synchronous orbit considered for Auroral Cluster is defined by a $500 \mathrm{~km}$ perigee altitude by a $6378 \mathrm{~km}\left(1 \mathrm{R}_{\mathrm{E}}\right)$ apogee altitude. For this orbit to be sun-synchronous the inclination must be fixed at $111.77^{\circ}$ to allow the nodal precession rate to match the average apparent solar precession rate of $.986^{\circ}$ per day. The $\Delta \mathrm{V}$ requirements for this orbit are the lowest of the three candidate orbits considered; thus this mission configuration is the least payload-mass-restrictive orbital option. The cluster's velocity in this orbit will be $8.68 \mathrm{~km} / \mathrm{s}$ at perigee and $4.68 \mathrm{~km} / \mathrm{s}$ at apogee. The initial argument of perigee would be $270^{\circ}$, establishing apogee and perigee above geographic latitudes of $68.23^{\circ}$ north and south respectively. Since this orbit is sun-synchronous it will have a constant solar illumination vector over the course of a year; a significant benefit in the design of the power generation system and the thermal management system. The apsidal rotation rate of $-.42^{\circ}$ per day will effect the mission in two ways: (1) the apogee will eventually rotate out of the auroral region and the cluster will not be traversing the regions of interest at the desired altitudes; but (2) given enough time ( -428 days), the apogee will have rotated $180^{\circ}$ and will occur at a geographic latitude of $68.23^{\circ}$ south, which will allow for sampling of the Earth's southern auroral regions.

\section{SSFLA Orbit}

The sun-synchronous, fixed line of apsides orbit possesses several properties that make it an ideal option for the AC mission. For the orbit to possess a fixed line of apsides and be sun-synchronous the inclination must be $116.6^{\circ}$. If a perigee altitude of $500 \mathrm{~km}$ is selected to minimize both launch vehicle requirements and atmospheric drag, then the apogee altitude must be $7903 \mathrm{~km}$ in order to obtain the sunsynchronous nodal rotation rate of $.986^{\circ}$ per day. The orbit's argument of perigee would be established at $270^{\circ}$, causing apogee to occur at $63.4^{\circ}$ north geographic latitude. The apogee altitude of $\sim 7,900 \mathrm{~km}$ is ideal since it coincides with the approximate center of the estimated auroral region's altitude range of 5,000 to $10,000 \mathrm{~km}$. The cluster's velocity in this orbit will be $8.84 \mathrm{~km} / \mathrm{s}$ at perigee and $4.26 \mathrm{~km} / \mathrm{s}$ at apogee. Of the three orbits considered, the SSFLA orbit allows for the smallest cluster velocity as it traverses the auroral regions.

The SSFLA orbit is an attractive option for the AC mission for a variety of reasons. Since the orbit is sun-synchronous, the solar flux vector remains constant over the mission lifetime and eases the design constraints on the thermal management and power generation subsystems. The sun-synchronous aspect of the orbit also allows the cluster to sample the same region of the magnetosphere at the same local time for each traversal. The fixed line of apsides aspect of the orbit assures that the cluster traverses the auroral regions at the same altitude during each traversal, and also assures that the apogee will not rotate out of the auroral regions as was the case for the HASS orbit discussed above. It should be pointed out, however, that since the Earth - and hence the magnetosphere - is rotating with respect to the cluster's orbit, neither the SSFLA nor the HASS orbit will traverse the auroral region during each orbit. The 
SSFLA orbit will, however, traverse some portion of the high-altitude auroral region during several orbits per day of the mission, whereas the HASS orbit will not traverse the high altitude auroral region every day of the mission because the orbit's apogee position will rotate out of the high geographic latitudes.

Table II Auroral Cluster Candidate Orbit Parameters

\begin{tabular}{|c|c|c|c|c|c|c|}
\hline Orbit & $\begin{array}{c}\text { Perigee } \\
\text { Altitude } \\
(\mathrm{km})\end{array}$ & $\begin{array}{c}\text { Apogee } \\
\text { Altitude } \\
(\mathrm{km})\end{array}$ & Inclination & $\begin{array}{l}\text { Period } \\
\text { (hours) }\end{array}$ & $\begin{array}{c}\mathrm{d} \omega / \mathrm{dt} \\
(\mathrm{deg} / \mathrm{day}) \\
\end{array}$ & $\begin{array}{c}\mathrm{d} \Omega / \mathrm{dt} \\
\text { (deg/day) }\end{array}$ \\
\hline HAHPP & 5,000 & 10,000 & $90^{\circ}$ & 4.52 & -.350 & 0 \\
\hline SSFLA & 500 & 7,903 & $116.6^{\circ}$ & 3.00 & 0 & .986 \\
\hline HASS & 500 & 6,378 & $111.77^{\circ}$ & 2.69 & -.415 & .986 \\
\hline
\end{tabular}

\section{Cluster Dynamics}

The success of the Auroral Cluster mission is highly dependent upon the ability to arrange the four spacecraft in a configuration that accommodates the gathering of data that can be used to derive the vector properties of $\mathbf{E}$ and $\mathbf{B}$. As stated in the mission requirements section, the desired arrangement for accomplishing this is to have each spacecraft positioned at the comer of a tetrahedron. Additionally, the interspacecraft range in the tetrahedron should be variable over the course of the mission from $10 \mathrm{~km}$ to $100 \mathrm{~km}$, i.e., the legs of the tetrahedron should be variable by an order of magnitude. To achieve a fixed tetrahedral configuration for the cluster at all times during the orbit is not possible without applying continuous thrust: an option well beyond the scope of this mission. It should be possible, however, to have the satellites obtain the desired tetrahedral configuration periodically, i.e., once per orbit. Of course the challenge is to establish, and then maintain, the proper initial conditions so that the cluster is in the tetrahedral configuration as it traverses the auroral region. To solve for these initial conditions one could employ the Clohessy-Wiltshire equations and apply them to the relative motion of the spacecraft in the cluster. ${ }^{3}$ The relative motion analysis for the $\mathrm{AC}$ mission has yet to be done; however, the significance of such an analysis has not been overlooked, and it represents one of the primary undertakings that should be executed in a more detailed AC mission study. For the purposes of this paper, however, it is possible to present a brief discussion of how the tetrahedral cluster can be periodically obtained.

The fundamental requirement for establishing the tetrahedral configuration is to place all four $\mathrm{AC}$ spacecraft in very similar but not identical orbits. One leg of the tetrahedron can be established by having two of the four spacecraft in identical orbits, but separated in the orbit plane by some difference in true anomaly. A third spacecraft would be in an orbit almost identical to that of the first two, with the only exceptions being a slight difference in inclination and a true anomaly that places it between the first two spacecraft when the two orbit planes intersect at their common line of nodes. The results of this arrangement will be to have the third spacecraft oscillating back-and-forth between the first two spacecraft during each orbit. This motion will create an oscillating triangular base of the tetrahedron, and will occasionally (twice in each orbit) create a three satellite "string of pearls" configuration where the two 
slightly different orbit planes intersect. The fourth spacecraft would be put into an orbit nearly identical to that of the first two, with the exception being a slightly different value of eccentricity. The difference in eccentricity will result in the fourth satellite oscillating in altitude with respect to the position of the two satellites in the first orbit. This motion will allow the fourth satellite to periodically occupy the apex point of the tetrahedron. All of the initial slight differences in the orbital elements for each satellite in the cluster can be established during satellite separation by applying some small $\Delta V$ relative to the other satellites. This $\Delta \mathrm{V}$ can be performed by either a spring ejection mechanism or a propulsion system in the satellite. To slowly expand the size of the tetrahedron out to $100 \mathrm{~km}$ over the course of the mission, and to provide station-keeping, will require a periodic $\Delta V$ of the satellites in the cluster. Thus, each satellite will need to have a small propulsion system capable of supporting the $\Delta \mathrm{V}$ manuevers.

\section{Launch Vehicle Considerations}

The ideal launch vehicle for the AC mission is the Delta II 7920 two-stage vehicle using the 2.9meter payload fairing. Although the Delta II is not typically used as a dedicated vehicle for small satellite missions, the vehicle is ideal for AC because its performance will allow four reasonably sized spacecraft to be deployed to any of the three candidate orbits with only one launch. Two of the technical arguments for launching all four satellites on one vehicle are (1) that the common launch assures that all initial orbital parameters are identical and (2) that the common launch eliminates the need to perform a complex rendezvous maneuver to configure the cluster on orbit. An additional advantage of the two-stage Delta is the ability to refire the liquid upper stage to trim the orbit to the desired orbital parameters before cluster deployment. The 2.9-meter payload fairing provides more than adequate volume to stack the four satellites and allows ample additional space for the many radially deploying appendages found on each spacecraft. The Delta II performance to the three orbits considered is: $973 \mathrm{~kg}$ to the HAHPP orbit, 1863 $\mathrm{kg}$ to the HASS orbit, and $1641 \mathrm{~kg}$ to the SSFLA orbit. ${ }^{4}$ Dividing any one of these mass numbers by four will yield the allowable individual wet spacecraft weight that can be delivered to the orbit of interest.

\section{STRAWMAN SCIENCE PAYLOAD}

The Auroral Cluster instrumentation is composed of an advanced set of representative sensors that will measure electric and magnetic fields, plasmas, and energetic particles. To fully resolve temporal and spatial features of auroral events, measurements will need to be made with a high degree of time resolution. This high time resolution requires that the instrument complement as well as the Command and Data Handling (C\&DH) subsystem be capable of very high data rates. For the strawman payload a data rate of approximately $1 \mathrm{Mbps}$ is required to meet the science objectives. Since it is impractical to support this data rate on a continuous basis, the instruments must be capable of both a low background data rate and a short-term high data rate burst mode. A third data taking mode, the "sleep" mode, where the scientific instruments will be turned off, will also be used to minimize telemetry, power, and memory requirements.

At pre-programmed times or latitudes of the orbit, the C\&DH subsystem will "wake up" the instruments and place them in the background data taking mode. Specific locations or times of auroral events of interest cannot be predetermined with sufficient accuracy to pre-program the high data rate intervals; thus the events must be identified in real time on the basis of in situ data. Each instrument will

have a pre-defined trigger level programmed into the C\&DH subsystem such that if that level is ever reached, the C\&DH subsystem will shift all of the scientific instruments into the burst mode. It is also important that all spacecraft capture high rate data simultaneously; thus event identification trigger 
commands must be broadcast between spacecraft. The instruments will stay in the burst mode until all instrument activity levels fall below their specified trigger levels. At that time, the C\&DH subsystem will again restore the instruments to the background data taking mode which will remain in effect until the sleep mode region of the orbit is entered..$^{5}$

Since precise correlative measurements will be made between different satellites, timing and synchronization of instruments, power supplies and C\&DH subsystems is mandatory. By using GPS timing signals, it should be possible to synchronize the Auroral Cluster spacecraft's clocks to within 100 ns of each other. 6

\section{Ion Measurements}

Two instruments will measure the ion plasma population. The Hot Plasma Composition Analyzer (HPCA), consisting of a toroidal top hat energy analyzer and a time-of-flight mass analyzer, will measure the high energy plasma particles. The cold plasma measurements will be made by a Cold Plasma Composition Analyzer (CPCA) such as the Toroidal Ion Dynamics Experiment (TIDE) currently being built for use on the ISTP POLAR spacecraft. The CPCA instrument uses a programmable electrostatic mirror and a retarding potential analyzer to perform the energy analysis of the ion spectrum and a time-offlight mass analyzer to determine the ion species. The time-of-flight portions of the HPCA and CPCA instruments could be identical. Table III lists many of the key characteristics of the HPCA and CPCA instrument. ${ }^{7,8}$ To save resources, the CPCA instrument will only be installed on two of the AC spacecraft.

Table II HPCA and CPCA Experiment

\begin{tabular}{ll}
\hline Configuration & $\begin{array}{r}\text { HPCA: Toroidal top hat energy analyzer w/time-of-flight } \\
\text { mass analysis }\end{array}$ \\
& CPCA: Electrostatic mirror/retarding potential analyzer w/time-of-flight \\
mass analysis
\end{tabular}


For the accurate measurement of cold plasma, the spacecraft potential with respect to the ambient environment must be maintained near the plasma potential. To actively control spacecraft charging, the satellite will be equipped with an ion emitter which will release indium ions to maintain the spacecraft charge at a neutral level. The ion emitter, which can emit a total current of $20 \mu \mathrm{A}$, will be hard-wired to the CPCA as well as to the electric wave experiment which will determine the amount of ion current that needs to be emitted to maintain the neutral spacecraft charge. The ion emitter will only be installed on the spacecraft that have a CPCA.?

\section{Plasma Wave Measurements}

The electric wave experiment will consist of two booms and four antennas. The booms will be 5 meter Weitzmann booms mounted parallel to the spin axis of the satellite. Each boom will have two $10 \mathrm{~cm}$ diameter sensors, one at the end of the boom at 5 meters and one at 4 meters (1 meter from the end of the boom). The four antennas will be positioned perpendicular to the spin axis of the satellite. Each of the antennae will consist of a radial wire 45 meters long and will again have two $10 \mathrm{~cm}$ sensors. The sensors will be placed 10 meters apart with one at the end of the antenna. The electric field experiment is very similar to that flown on Viking and Freja. ${ }^{10,11,12}$ Potential difference will be measured between opposite booms, between adjacent booms, and between the sensors on the same boom. The electric wave experiment is vital for the mission as it will measure the plasma density, the $\mathrm{K}$-vector, and polarization of the plasma environment. Key characteristics of the electric wave experiment are listed in the table IV.

Table IV Electric Fields and Waves Experiment

\begin{tabular}{ll}
\hline \hline & \\
Configuration & $\begin{array}{l}4 \text { radially mounted wire antenna, each } 45 \mathrm{~m} \text { long, each } w / 210 \mathrm{~cm} \text { dia } \\
\text { sensors located } 10 \mathrm{~m} \text { apart } \\
2 \text { axially mounted rigid booms, each } 5 \mathrm{~m} \text { long, each } w / 210 \mathrm{~cm} \text { dia sensors } \\
\text { located } 1 \mathrm{~m} \text { apart }\end{array}$ \\
& E, DC-10 MHz, total electron density, K vector, polarization \\
Measurement & Background mode $=40 \mathrm{kbits} / \mathrm{s} ;$ Burst mode $=400 \mathrm{kbits} / \mathrm{s}$ \\
Telemetry & $7.0 \mathrm{~kg}$ for electronics and sensors; \\
Mass & $25 \mathrm{~kg}$ for total experiment \\
& 15 watts \\
\hline
\end{tabular}

\section{Electron Measurements}

The electron energy spectrum will be measured by two electron spectrometers. The spectrometers will consist of a spherical top hat energy analyzer and a micro-channel plate anode system. Key characteristics of the experiment are listed in Table V. The spectrometers will share a common data processing unit but will serve different functions. The top hat detector mounted with its acceptance "plane" parallel to the orbit plane will serve to provide very high-time-resolution energy spectra. An 
elevation analyzer will be mounted before the energy analyzer to increase the angular acceptance of the instrument from $5^{\circ} \times 360^{\circ}$ to $90^{\circ} \times 360^{\circ}$. The magnetometer located on the satellite will determine the position of the magnetic field in relation to the satellite in real time. With this knowledge the data processing unit for the electron spectrometer will adjust the voltage of the elevation analyzer to force the acceptance plane of the spectrometer to be in the plane of the magnetic field vector.

The electron spectrometer, whose axis of symmetry is perpendicular to the spin axis of the spacecraft, will take advantage of the spin to obtain full $4 \pi$ coverage of the environment. The two spectrometers will be identical except for the elevation analyzer attached to the front end of the high-timeresolution instrument. The top hat detector is a proven design with much flight history. ${ }^{13}$

Table V Electron Energy Spectrometer

\begin{tabular}{|c|c|}
\hline Configuration & $\begin{array}{l}2 \text { top hat analyzers; A) one deployed radially, B) } \\
\text { sensor's axis of symmetry is parallel with the spa } \\
\text { Sensor B is equipped with an electrostatic elevati }\end{array}$ \\
\hline Field of View & $\begin{array}{l}\text { Sensor A: } \\
\text { Static: } 360^{\circ} \times 5^{\circ} \text { fan } \\
\text { With spacecraft spin: full } 4 \pi \text { coverage } \\
\text { Sensor B: } \\
\text { Static: } 360^{\circ} \times 5^{\circ} \text { fan } \\
\text { With elevation analyzer: } 360^{\circ} \times 90^{\circ} \text { fan }\end{array}$ \\
\hline Energy Range & $10 \mathrm{eV}$ to $90 \mathrm{KeV}, 10 \%$ energy resolution $(\Delta \mathrm{E} / \mathrm{E})$ \\
\hline Geometric Factor & $1.17 \times 10^{-3} \mathrm{~cm}^{2} \mathrm{sr}$ per pixel $\left(3610^{\circ}\right.$ pixels $)$ \\
\hline Telemetry & $\begin{array}{l}\text { Background mode }=10 \mathrm{kbits} / \mathrm{sec} \text { each; } \\
\text { Burst mode }=100 \mathrm{kbits} / \mathrm{sec} \text { each }\end{array}$ \\
\hline Mass & $10 \mathrm{~kg}$ total \\
\hline Power & 10 watts total \\
\hline
\end{tabular}

\section{Auroral Imaging}

Two of the satellites will also be equipped with imagers. The baseline imager for the mission is the ultraviolet auroral imager that was used on the Viking spacecraft. The imager consists of two intensified wide-angle $\mathrm{f} / 1$ cameras. They are identical except in passband with electronic despinning providing exposure time of .15 second. The imagers take advantage of tapered fiber optic bundles to remove distortions that are generated when the spherical focal surface of the Burch configuration camera is projected onto the plane of the charge coupled detector. ${ }^{14}$ By equipping two of the satellites with imagers, not only is redundancy gained but also stereoscopic imaging of the aurora is now possible. Key characteristics of the imager can be found in Table VI. 
Table VI Auroral Imaging Experiment

\begin{tabular}{ll}
\hline \hline & \\
Configuration & Sensor composed of $2 \mathrm{f} / 1$ cameras; $1240-1500 \AA$ and $1300-1900 \AA$ \\
Field of View & $29^{\circ} \times 24^{\circ}$ \\
Image Speed & 1 image per spin $(.15 \mathrm{sec}$ exposure time $)$ \\
Telemetry & Background mode $=10 \mathrm{kbits} / \mathrm{sec} ;$ \\
& Burst mode $=100 \mathrm{kbits} / \mathrm{sec}$ \\
Mass & $8.5 \mathrm{~kg}$ \\
Power & $5.3 \mathrm{~W}$
\end{tabular}

\section{Magnetometer}

The final experiment of the Auroral Cluster program is the Magnetic Field experiment, which will consist of a three-axis fluxgate magnetometer mounted on a 5-meter rigid boom. The system has heritage to the Viking mission as well as numerous other flights. ${ }^{10}$ The magnetometer will function within four ranges from $\pm 1024( \pm 0.125)$ nT to $\pm 65,536( \pm 8) n T$. Table VII gives key characteristics of the Magnetic Field Experiment.

Table VII Magnetic Field Experiment

\begin{tabular}{ll}
\hline \hline & \\
Configuration & 3-axis fluxgate magnetometer on $5 \mathrm{~m}$ long rigid boom \\
Measurement Range & $\pm 1024( \pm 125) \mathrm{nT}$ to $\pm 65,536( \pm 8) \mathrm{nT}$ \\
Sample Speed & 53 samples per second \\
Resolution & $\geq 6 \mathrm{pT}$ \\
Telemetry & $2.2 \mathrm{kbits} / \mathrm{s}$ \\
Mass & $2.1 \mathrm{~kg}$ \\
Power & $1.1 \mathrm{~W}$ \\
\end{tabular}

\section{SPACECRAFT CONSIDERATIONS}

The baseline configuration for the Auroral Cluster mission consists of four spacecraft that are spin stabilized with their spin axis normal to the orbit plane. Two of the four spacecraft are labeled as "mother" vehicles, because of their increased complement of science instruments, and because they provide 
the cluster's RF Earth link. The remaining two spacecraft are labeled "daughter" vehicles because of their smaller instrument complement and because they depend upon the "mother" vehicles to downlink their telemetry. Each spacecraft is expected to carry approximately $60 \mathrm{~kg}$ of science payload. The design goals for each spacecraft are to have a wet mass less than $400 \mathrm{~kg}$, generate a minimum of $-200 \mathrm{~W}$ continuous power, determine attitude to $.01^{\circ}$ and control attitude to $1^{\circ}$, and be capable of operating for a one year mission life. At this early stage of the AC mission design process, it is impossible to present a detailed and accurate spacecraft design. It is possible, however, to discuss some of the design options that exist for the subsystems that will make up the AC spacecraft. Although not intended to be an exhaustive presentation of all options, the following discussion serves as a brief introduction to some of the trade-offs that will need to be addressed before the detailed design of the AC spacecraft begins.

\section{Position Determination}

The AC science requirements demand that the intersatellite (relative) position of each satellite be known to 100 meters or within $1 \%$ of the cluster's intersatellite separation distance during auroral region traversals. The absolute position of the cluster in an Earth-centered frame must also be known to within $10 \%$ of the cluster's size, i.e., if the bases of the tetrahedron formed by the cluster are $10 \mathrm{~km}$, then the absolute position of the cluster should be known to within $1 \mathrm{~km}$. The ideal and most autonomous way to obtain this knowledge is to employ the capabilities of the Global Positioning System (GPS).

GPS offers a new, very precise, and multipurpose system for determining the position of a spacecraft in low Earth orbit (LEO). A variety of upcoming missions - most notably the TOPEX/POSEIDEN and the EOS programs - will be flown with onboard GPS receivers to demonstrate the system's ability to precisely determine a satellite's position in low-altitude orbits. The position determination capabilities made available by the GPS system are remarkable; TOPEX is anticipating the ability to determine the satellite's altitude to a sub-decimeter accuracy by employing differential GPS. ${ }^{15}$ Although sub-decimeter position knowledge is not required for $\mathrm{AC}$, position knowledge accuracy on the order of one to ten meters is desirable. Therefore, the combination of GPS' capabilities makes its use ideal for solving a variety of the AC mission problems, which include the precise determination of absolute and relative spacecraft position, the determination of spacecraft attitude, and the ability to provide very accurate clock synchronization for all the spacecraft and instruments in the cluster.

Employing GPS for the AC mission, however, is not as straightforward as it is for spacecraft residing in LEO. The AC mission's high-altitude excursions into the auroral regions will result in the cluster departing from spatial regions that are normally illuminated by the Earth-pointing beams of the GPS constellation; thus, traditional "up-looking" differential GPS is not always functional for the spacecraft in the proposed AC orbits. There are, however, two altemative GPS methodologies that may work: (1) "down-looking" differential GPS and (2) "inverted" differential GPS ${ }^{16}$. Obtaining the desired relative and absolute position knowledge of the AC spacecraft using these alternative GPS methods appears to be a feasible option. By employing the "down-looking" differential GPS technique, and supporting the AC mission with a GPS ground station network (being developed to support the variety of upcoming GPS spaceborne user missions), it appears possible to obtain absolute AC spacecraft position in the 1 to 10 meter range. ${ }^{15,16}$. If the AC cluster is to implement the GPS capabilities, a multichannel, differential-capable GPS receiver, as well as isotropic GPS antennas must be flown on each spacecraft. As a baseline system to support the early AC spacecraft sizing exercise, we have used the mass and power numbers for Motorola's Monarch Spacebome GPS user receiver. This system weighs $4 \mathrm{~kg}$ and is expected to consume 30 watts. ${ }^{17}$ 


\section{Attitude Determination and Control}

Much of the success of the AC program will depend on the ability to determine the precise attitude of each spacecraft in the cluster. As explained earlier, the attitude of each spacecraft must be determined to within $.01^{\circ}$, a level of accuracy seldom, if ever, required on previous space physics missions. The selection of the attitude control configuration for the mission is reduced to a trivial exercise, since a 20 $\mathrm{rpm}\left(120^{\circ}\right.$ per second) spin-stabilized configuration is necessitated by the need to obtain the threedimensional high-time-resolution plasma environment data. Unfortunately, the combination of the spacecraft's relatively high spin-rate control configuration with the need to determine attitude to within $.01^{\circ}$ represents a significant technical challenge that must be overcome in order to implement the AC mission.

\section{Attitude Determination}

Traditionally, a $.01^{\circ}$ level of attitude determination accuracy would be obtained by employing some form of star detecting device, e.g., a star scanner, star mapper, or star tracker. For a spin-stabilized spacecraft, such as the AC spacecraft, the star scanner would be the instrument of choice for obtaining $.01^{\circ}$ attitude knowledge. For a variety of reasons, however, a star scanning device may not represent the ideal technology for the AC mission. Some of the specific reasons for wanting to exclude a star scanner from use on the AC mission include: (1) star scanners tend to be massive, and voluminous, and they consume considerable amounts of power, (2) there do not appear to be any star scanners in production or design at the present time; and (3) modifying an old star scanner design or developing a new star scanner design capable of functioning on a spacecraft spinning at $20 \mathrm{rpm}$ would entail a significant and costly development effort. The conclusion, therefore, is that star scanners represent a costly technology that could work, but if a functional alternative exists then it might be worth pursuing.

The primary alternative to the use of a star scanner would be to employ the capabilities of the GPS system. This is an especially attractive option for the AC mission because some of the necessary hardware infrastructure will already be in place if GPS is used to determine the position of each spacecraft in the cluster. The direct approach to using GPS for determining a spacecraft's attitude entails the use of interferometric techniques on the signals transmitted by the GPS space vehicles. A considerable amount of work has been done to demonstrate the feasibility of this approach. ${ }^{6,18,19,20}$ By employing two GPS antennas separated by a baseline of length $\mathrm{L}$, and by interferometrically combining the signals received at the two antennas, it is possible to determine the attitude of the baseline. ${ }^{6}$ If three different baselines are used, it is possible to determine the attitude of a vehicle about three axes. It is estimated that the precision of such measurements can be made to below the desired $.01^{\circ}$, provided the system is configured properly; in fact, the Naval Research Laboratory is managing the Space Navigation and Pointing System (SNAP) program that hopes to be able to demonstrate 1 urad attitude determination capability using GPS. ${ }^{18}$ Although a variety of factors determine the accuracy of the measurement, e.g., system noise, dilution of precision, integration times, etc., one of the significant issues that will affect the ability to use GPS for attitude measurements on AC will be the ability to place the GPS antennas at a great enough distance apart $(\mathrm{L})$ to obtain an attitude measurement of the desired accuracy. If a $.01^{\circ}$ attitude knowledge is desired, then the antenna separation distance, $\mathrm{L}$, could be as small as 1 to 5 meters for the properly configured system. ${ }^{6,20}$ By deploying two isotropic GPS antennas on the axially mounted plasma wave experiment booms, it should be possible to obtain a baseline length of anywhere from 2 to 5 meters. Although such a configuration will only yield one attitude vector via GPS, a narrow field-of-view, highaccuracy sun sensor could also be employed to fully resolve the vehicle's attitude. 


\section{Attitude Control}

The AC spacecraft will be spin stabilized at $20 \mathrm{rpm}$. This configuration is optimum for allowing the science instruments to monitor the plasma's 3-dimensional properties with high time resolution. The additional benefits of using a spin stabilized design are: it minimizes the mass of the attitude control subsystem, it represents an approach that can be readily implemented and maintained, and it is a very efficient control technique for a mission like $\mathrm{AC}$ that does not require periodic slewing maneuvers. Initial spacecraft spin-up can be achieved by the launch vehicle's upper stage or by firing vernier thrusters. A spin control system will be required, and could consist of an appropriately configured arrangement of thrusters and a nutation damper. To minimize nutation, proper and precise balancing of the spacecraft will be mandatory. A significant amount of analysis to model, predict, and compensate for the dynamics of the radially deployed booms will be required.

\section{Command and Data Handling Subsystem}

The Auroral Cluster Command and Data Handling (C\&DH) subsystem is composed of two main functional blocks, a Central Processing Unit (CPU) and a mass storage unit. Two different architectures distributed and centralized, exist for satellite command and data handling subsystems. Multiple-satellite missions such as Auroral Cluster provide yet another choice, distributing the C\&DH system between satellites using electronic tethering. Further details about this intersatellite distributed system will be given in the following sections.

The requirements of the command and data handling subsystem are: ${ }^{21}$

1. Interface to the Communication and the Attitude Determination \& Control subsystems.

2. Provide on-board temporary mass data storage.

3. Receive and issue commands for satellite and instrument mode changes or status changes.

4. Run "standard" programs for control of the satellite and instruments.

5. Provide on board processing, formatting, compressing and combining of data.

6. Monitor the status of the satellite and instruments for health and safety.

\section{Instrument Interface}

The first interfaces that the C\&DH subsystem must connect to are the instrument interfaces. Again, the question of where or how to distribute the system memory and data processing arises. One option, the distributed systems approach, uses "smart instruments" that have their own mass memory storage and microprocessors. This intelligence at the instrument level makes the commands and data that are transferred between the instrument and the C\&DH subsystem relatively simple. Cabling is reduced, interfaces are simpler, and the possibility of noise pickup is reduced.

The other extreme, a centralized C\&DH system or nondistributed system, uses "dumb" instruments. With centralized systems, very little mass storage is available to the instrument at the instrument location. The instruments typically do not have microprocessors, and the commands and data that are shifted back and forth on the bus tend to be more complicated as well as in larger quantities. Advantages of centralized systems include simpler instrument design; economies of scale concerning storage, processing and compressing of data; and more control by the spacecraft. There are many tradeoffs and advantages for each of these different systems. 


\section{Spacecraft Interface}

The second interface that the command and data handling subsystem must be able to coordinate is the spacecraft interface. The role of the C\&DH subsystem when dealing with the spacecraft system is one of monitoring and issuing commands. In many cases the monitors are analog in contrast to the instrument interface where the lines are typically digital. The command and data handling system will monitor temperatures, voltages, currents and limit switches. The C\&DH subsystem will command the different operations of the satellite, such as boom deployments, and any kind of attitude or positional adjustment that the satellite needs to perform.

Again, the amount of satellite autonomy is a question which must be addressed. Processing power can be distributed among the cluster of satellites or can be placed on one or two of the satellites and shared between the four satellites. A detailed study of these different trade-offs, which is beyond the scope of this paper, should be undertaken. The study must focus on cost, weight, power and other factors at a mission level.

\section{GPS Receiver Interface}

The GPS receiver, which is part of the Attitude Determination and Control subsystem, must also interface with the C\&DH subsystem. The GPS receiver will provide many important pieces of information to the C\&DH subsystem, including position and attitude determination of the satellite and a clock and timing signal. The timing signal will be used to synchronize all power supplies in the cluster to the same frequency, thus eliminating unwanted noise at random frequencies and making possible multipoint correlated wave-particle measurements. This synchronization is particularly important for the Electric Fields and Waves experiment ability to perform interferometric measurements. Specific details and specifications of the GPS receiver capabilities are found in the Attitude Determination and Control subsystem section.

\section{Data Storage}

There are three mass data storage options for the AC mission. The first option is to place all the memory at the instrument level. The instrumenters would likely be responsible for the purchase, design, fabrication and test of this memory. The second option, a centralized mass storage system per satellite, utilizes a single mass storage device on each satellite to which all instruments send their data. For the strawman list of instruments memory required for one orbit per satellite is approximately 200 Mbytes. The final option for the distribution of the mass storage is using one or possibly two centralized mass storage units for all four satellites. A possible scenario could be two "mother" satellites equipped with mass storage units, and two "daughter" satellites that would transmit all of their data to the mother for storage until the time to downlink the data. The advantages of this option include the elimination of the weight, power, and cost of separate data storage units on two of the satellites, while still retaining redundancy.

In today's technology, three options exist for large mass storage devices: Random Access Memory (RAM) chips; tape recorders, which have been successfully used for many years on numerous missions; and new, emerging technologies such as optical disks or magnetic disks. These new technologies are presently in the breadboard stage, but are expected to be space-proven within the next year or two. At the time of spacecraft design, a detailed trade-off study should be performed to find out what is the most cost-effective and reliable method to store a large quantity of data. At present in the storage capacities needed, RAM costs approximately $\$ 20 \mathrm{k}$ per megabyte, tape recorders cost approximately $\$ .5 \mathrm{k}$ per megabyte, and optical disk drives, though only in the breadboard stage are expected to cost approximately 
$\$ .3 \mathrm{k}$ per megabyte. ${ }^{22,23}$ Obviously, all of these costs will change within the next few years and should be carefully looked at when the mission is being designed.

\section{Communication Subsystem Interface}

The Communication subsystem is the subsystem that is responsible for sending and receiving data and commands away from and to the satellite. Other important pieces of information that will have to be transmitted or received include clock timing signals, position and attitude data, and health and safety checks. One of the Command and Data Handling subsystem functions is to format, combine, compress, and process the data into a form where the Communication subsystem can further transmit or receive it. In addition to data processing, these tasks will be the main function of the Central Processing Unit.

\section{Data Processing}

When one designs a satellite, one always faces the question of where best to perform the data processing, on-board or on the ground. The drastic cut in the amount of telemetry to be sent down to the ground each orbit is the key reason for performing data processing on board. However, on-board processing complicates the design of the satellite, increases the complexity of the command and data handling subsystem, and typically increases weight, power, and cost of the satellite. The advantage of doing the processing on the ground is unlimited, inexpensive power and computing. Large mainframe computers can be used for data processing in a non-rushed mode. The principle disadvantage to processing on the ground is, as stated, the large amount of telemetry and data which one receives and possibly does not need.

With the Auroral Cluster mission, there is an additional choice: perform the data processing on board one satellite or on board each satellite. A detailed trade-off study should be performed to establish the optimum amount of processing and the location of this processing for the AC mission.

Other considerations besides cost, weight, and power must be factored into the data processing decision. One such factor is redundancy and reliability. Again, a "mother-daughter" scenario could be used: the mother satellites would not only provide the mass storage, but would also provide all of the onboard processing. The system would be configured such that if either mother failed the other would act as a backup and take over.

\section{Command \& Data Handling Subsystem Tradeoffs}

The design of the C\&DH subsystem contains more options than any other subsystem of the AC mission. A detailed mission utility analysis needs to be performed to determine the optimum configuration for the subsystem. Easily quantifiable factors that must be examined are how the different options affect program resources such as mass, cost, volume, and power. Other factors of at least equal importance that also need to be examined include system reliability and redundancy, required test and integration time, amount of needed program coordination, ability to meet the mission goals, system adaptability, system risk, and the potential engineering knowledge gained by the technology. The mission utility analysis is one the first steps in the implementation of the Auroral Cluster program.

\section{Communication Subsystem}

The Auroral Cluster Communication subsystem provides the link between the satellite and Earth and the satellite and the other AC satellites. The Communication subsystem interfaces to the Command and Data Handling subsystem, which directly controls the instruments and the spacecraft itself. ${ }^{21}$ 
Requirements of the Auroral Cluster communication system are:

Command reception and detection

- $\quad$ acquire, track, and demodulate uplink carrier

- $\quad$ derive bit timing and detect data bits

- $\quad$ receive commands and signals from other Auroral Cluster satellites

Telemetry modulation and transmission

- $\quad$ receive telemetry data stream from $C \& D H$ subsystem

- $\quad$ modulate downlink carrier with mission and science telemetry

- transmit composite signal (i.e., data, commands, clock, position, attitude, mode changes and commands) to Earth (or relay satellite such as TDRSS) and other AC satellites.

Subsystem operations

- $\quad$ receive commands from the command and data handling subsystem

- $\quad$ provide health and status telemetry to the C\&DH subsystem

- autonomously detect faults and recover communications using stored software sequence

As stated in the strawman payload instrument section of this paper, the Auroral Cluster mission will utilize three different data taking modes, depending on location of the cluster in orbit, and also dependent on the amount of activity in the plasma environment. The three modes of data-taking are the burst mode, the background mode, and the sleep mode. If fifteen minutes of data-taking in the burst mode and one hour of data-taking in the background mode are assumed, each satellite will accumulate approximately 200 megabytes of telemetry per orbit including health and safety monitors and housekeeping data. This translates into 800 megabytes of data for the entire $\mathrm{AC}$ satellite complement per orbit. This amount of data will need to be telemetered to the ground at least once per orbit.

Just as the Command and Data Handling subsystem has multiple options, so too does the design of the Communication subsystem offer options that must be considered. Under the first option, each satellite would be required to downlink its own data to the ground. Each satellite would be autonomous and create its own data stream. Each satellite would still need to be able to communicate with the other members of the cluster.

A second option would be to use the "mother-daughter" scenario discussed above. The two mother satellites would be responsible for downlinking the data of the entire cluster. By having two mothers, the telemetry system would be redundant. One possible method of implementing and monitoring the health of the redundant telemetry system would be to switch every other orbit which of the two mother satellites transmits the data to the ground.

By requiring that only two of the four satellites have the ability to transmit and receive data to and from the ground, it might be possible to use smaller, lower power transponders on the daughter payloads. Also receiving and transmitting only one data stream rather than four will place fewer demands on the ground station. Since GPS receivers will be utilized on each of the four satellites, position and attitude of the satellites will be known to a very high accuracy, so the traditional problem of tracking and ranging should be eliminated.

With the AC mission, two different communication links exist. The first of these is between satellites. The second communication link is between the cluster and Earth. Both of these links have advantages and disadvantages for the design of an overall communication system. The advantage of the satellite-to-satellite communication link is the short path distance involved. The maximum separation distance is on the order of 300 kilometers. The advantage of the satellite-to-Earth or Earth-to-satellite 
communication link is that it is very easy and cost-effective to use large high-gain antennas on Earth to send or receive signals to the constellation. The overall Communication subsystem must be designed to minimize on-board weight and power requirements.

Another complication that the $\mathrm{AC}$ mission requirements place on the Communication subsystem is that attitude control is accomplished by using spin stabilization, rather than utilizing a three axis stabilized platform. Three-axis stabilized platforms are always easier for Communication subsystems because directional antennas can easily be pointed accurately. On spin-stabilized satellites, such as the Auroral Cluster satellites, a despun platform would be required to obtain this same antenna-pointing accuracy. For the AC mission the cost and complexity that come with the use of a despun platform are beyond the scope of the mission. Preliminary calculations show that two sets of omnidirectional $90^{\circ}$ opposed short dipole antennas can be used on each satellite. The antennas would be designed to have a bidirectional cosine power pattern. This pattern should provide ample coverage for sending information between satellites and also for sending data from the satellites to the Earth.

Preliminary communication link calculations show that for the satellite-to-satellite link, S-band is nearly optimum. This frequency provides enough bandwidth for the telemetry requirements. Downlink time per orbit using this frequency is roughly 10 minutes. Obviously, a detailed study of the communication system must be performed after a final orbit is selected and after a better definition of telemetry requirements has been obtained.

\section{Power Subsystem}

The requirements imposed on the power subsystem for the $\mathrm{AC}$ mission are not unlike those that have been successfully met in many previous spaceflight programs. Some of these specific requirements for the AC configuration described here include: the generation of an anticipated minimum of 200 watts of continuous power throughout the mission, the regulation and distribution of power on a low noise 28 volt bus, and the storage of adequate power for use during eclipse or to augment the solar arrays when peak loads exceed the array's nominal power output. Based upon the success of past programs with similar requirements, it is reasonable to expect that these requirements can be met by the use of existing, spaceflight-proven technologies. The challenge, however, will be to achieve the design of an adequate power system, while minimizing the mass and budget resources of the system. Since solar arrays and batteries represent a significant fraction of the mass and cost of the total power subsystem, the possible trade-offs associated with them merit a brief discussion.

\section{Solar Cells/Arrays}

The spin stabilized configuration of the AC spacecraft mandates that the solar arrays be distributed about the spacecraft's circumference and possibly on either of the two ends; the latter arrangement being dependent upon the cluster orbit chosen. The array performance, measured in output wattage, will depend upon a variety of factors, some of which are: active array size (highly dependent on spacecraft form factor), array temperature, array pointing with respect to the sun, and individual cell efficiency. Cell efficiency is usually dictated by the technology employed in fabricating the cell. Current technology narrows the selection of cells down to a choice between silicon or gallium arsenide cells. Silicon cell technology provides for flight worthy cells with efficiencies on the order of $15 \%$. In contrast, GaAs cells can achieve an efficiency on the order of $17 \%$, although at a significantly greater cost than that of silicon cells. ${ }^{24}$ The tradeoff is a straight-forward comparison of cost, mass, and performance issues. It is currently anticipated, however, that the goals of the AC program can best be met by employing the lower cost silicon cell option. Should the mission duration be extended considerably, or if the mission's mass 
budget becomes very restricted, it might become necessary to reexamine the option of using GaAs cells.

\section{Batteries}

The present battery technologies under consideration for the $\mathrm{AC}$ mission include nickel-hydrogen $\left(\mathrm{NiH}_{2}\right)$, nickel metal hydride, and nickel cadmium. The $\mathrm{NiH}_{2}$ battery configuration would provide a lightweight, high-efficiency system, but at a greater cost than the latter two options. Since the true battery requirements are not yet fully determined, it is difficult to assess which design offers the best approach. Given the intended mission duration of only one year, however, it is reasonable to expect that a NiCd configuration will represent the lowest cost if the mission mass budget is not overly constrained.

\section{Propulsion Subsystem}

The propulsion requirements for the $\mathrm{AC}$ mission consist primarily of supporting the attitude control subsystem and providing the necessary $\Delta \mathrm{V}$ to adjust the cluster's configuration over the course of the mission. If the launch vehicle is capable of injecting the cluster directly into the working orbit, then the $\mathrm{AC}$ spacecraft will not need to perform major, high impulse $\Delta \mathrm{V}$ maneuvers. Although there are typically three choices when selecting an onboard propulsion system - cold gas, monopropellant, or bipropellant - the requirements for the $\mathrm{AC}$ mission are small enough that only cold gas and monopropellant are considered real options. Of these two remaining options, the monopropellant configuration is considered the best option. The reasons for proposing the monopropellant system include the higher specific impulse capabilities of such a system, and the ready availability of hardware. A single stage blowdown system using hydrazine will allow the propulsion system to support both the attitude control system and provide the necessary $\Delta \mathrm{V}$ for cluster expansion. The exact sizing of the propulsion system will proceed after the final orbit for the $\mathrm{AC}$ mission is chosen.

\section{Thermal Design}

The design of the Auroral Cluster spacecraft will maximize the use of conventional passive thermal management techniques. When necessary, electronics and sensors will be thermally isolated from the body of the spacecraft with specifically designed mountings and multilayer insulation blankets. Radiative surfaces will control the temperature of the analyzers and electronics. Flexible resistive heating elements will provide heating if necessary at instrument turn on and for periods of the orbit when the spacecraft is eclipsed with the instrument power off.

An analytical model needs to be made for the spacecraft, including booms and antennas, once the final orbit parameters are selected. The model will consider the orbital environments that the spacecraft will experience and will include all shields, insulation, radiative surfaces, and heaters. The model will have enough fidelity to predict temperature extremes for all critical surfaces and parts. The thermal design of the AC mission is straightforward and presents no new challenges to the system design engineer.

\section{Structure, Mechanisms, and Configuration}

The structures and mechanisms subsystem provides the common mechanical support for all the hardware that comprises the other subsystems and the instruments. In addition, the system provides both the structural load path for distributing the launch loads to the launch vehicle interface, and the hardware for ordinance-activated spacecraft separation. The system does not employ the use of moving devices, motors, despun platforms, or other hardware that traditionally complicates the system mechanical design. The booms and antennas will be "off the shelf" flight proven designs that will integrate easily with the 
spacecraft's structure. Mechanically, the most complicated system is expected to be the deployment hardware for initial spacecraft release and cluster deployment.

The structure of the AC spacecraft will not be unlike the structural configuration used on previous spin-stabilized spacecraft. The baseline form factor of the AC spacecraft is a cylinder with a height of .5-meters and a diameter of 1.5-meters. The two circular deckplates forming the ends of the cylinder, will serve as mounting platforms for the various payload electronics boxes. A single tube or cone will run axially along the center of the cylinder, and serves as the primary load carrying element. Bulkheads will radiate from the central tube, and will provide the primary load path from the spacecraft's external structure into the central tube. Solar arrays will attach to the circumference of the spacecraft with stringers. The center of the spacecraft will contain the propellant tankage and the various other subsystem hardware. The design of the AC spacecraft's structure will strive to maximize integrity while minimizing structural weight.

The sensitivity of the scientific instrument complement will mandate the use of specialized materials and coatings. When possible, time proven spaceflight materials will be used in the construction of all sensors and structures. Magnetic cleanliness will be adhered to as much as possible. To keep the overall magnetic field of the AC spacecraft to a minimum, AC will use only magnetically clean material. All materials used will be drawn from NASA Reference Publication 1124 and will have a Total Molecular Loss of less than $1.0 \%$ and a Collected Volatile Condensed Mass of no more than $0.10 \%$. The particle instruments impose this outgassing requirement. Exposed dielectrics and potentials will be minimized. To minimize EMI/EMF, the cabling plan of the AC spacecraft will use a star-ground and be designed to eliminate potential ground loops. These stringent requirements are required by the electric fields and wave experiment.

\section{CONCLUSIONS}

Auroral Cluster is a space physics mission of significant scientific merit, which will enhance our knowledge of the Earth's auroral regions and help us to further understand the coupling between the solar wind and the magnetosphere. This paper has identified many of the primary science mission requirements and demonstrated how these requirements manifest themselves as spacecraft subsystem requirements. It appears to be feasible to employ four small satellites that are "electronically tethered" to achieve the AC mission goals. Based upon the discussion of the possible subsystem trade-offs, it is reasonable to believe the AC mission could be implemented with existing "off the shelf" hardware. The next logical step in furthering the design of the AC program would be to conduct a mission utility analysis that would examine all possible design trade-offs and terminate with a phase A mission design study.

Although the scope of the AC mission described in this paper is appropriate for a typical NASA Delta Class Explorer program, recent discussions within the scientific community have resulted in AC's being recommended for a new start as one of NASA's new Medium Class Explorer programs. To implement the AC program presented here as a Medium Class Explorer is highly feasible, provided that some descoping of science instrumentation be made, and the use of a launch vehicle smaller than the Delta II be considered. Even for a descoped mission, however, the basic engineering challenges and trade-offs remain the same as those discussed here. 


\section{Acknowledgements}

The writing of this multi-disciplined paper would not have been possible without the guidance and help of many individuals. We would especially like to thank P. Kintner, B. Sandel, D. King and R. Winglee for their technical and scientific expertise, G. Perez for typing assistance and $W$. Lewis for his writing and proof reading skills.

\section{References}

${ }^{1}$ Dynamics Explorer, Edited by R.A. Hoffman, D. Reidel Publishing Company, Dordrecht, Holland, 1981. ${ }^{2}$ Space Physics Strategy - Implementation Study, Volume 1: Goals, Objectives, Strategy, Report of Workshop I, Baltimore, Maryland, 1991 and Space Physics Strategy - Implementation Study, Volume 2: Program Plana, Report of Workshop 2, Bethesda, Maryland, 1991.

${ }^{3}$ Wiesel, W.E., Spaceflight Dynamics, McGraw Hill Book Company, 1989, pp. 78-83.

${ }^{4}$ Private communication with Commercial Delta II Marketing Representative, Wednesday, July 31, 1991.

5"Magnetospheric Multiprobes," Preliminary Mission and System Description, prepared by Southwest Research Institute, San Antonio, Texas, 1983.

"Spacecraft Applications of Advanced Global Positioning System Technology," Final Report for Contract No. NAS9-17681, Axiomatix Tau Corporation Motorola, Inc., 1988.

${ }^{7}$ Young, D.T., J.A. Marshall, J.L. Burch, "A $360^{\circ}$ Field-Of-View Toroidal Ion Composition Analyzer Using Time-Of-Flight," Solar System Plasma Physics, Geophysical Monograph, 54, 171, 1989.

${ }^{8}$ Young, D.T., "Space Plasma Mass Spectroscopy Below $60 \mathrm{keV}$," Solar System Plasma Physics, Geophysical Monograph, 54, 143, 1989.

${ }^{9}$ Schmidt, R., and M.L. Goldstein, "Cluster - A Fleet of Four Spacecraft to Study Plasma Structures in Three Dimensions," ESA Publications Division, ESA SP-1103, 1988.

${ }^{10}$ Hultqvist, B., "The Viking Project," Geophys. Res. Letts., 14, 4, 1987.

${ }^{11}$ 5.5-Meter Tip-to-Tip E-Field Experiment Boom, Mechanism with Ejectable Booms, Specification No. 78200, Weitzmann Consulting, Inc., San Francisco, California, 1986.

${ }^{12}$ Interface Control Radial Wire Boom System 84100 (Freja-WB1 through WB6), Drawing Number 84100-DICD, Rev. D, for Weitzmann Consulting, Inc., San Francisco, Califomia, 1985.

${ }^{13}$ Sharber, J.R., J.D Winningham, J.R. Scherrer, M.J. Sablik, C.A. Bargainer, P.A. Jensen, B.J. Mask, N. Eaker and J.C. Biard, "Design, Construction, and Laboratory Calibration of the Angle Resolving Energy Analyzer (AREA): A "Top Hat" Instrument for Auroral Research," IEEE Trans. on Geoscience and Remote Sensing, 26, No. $4,1988$.

${ }^{14}$ Anger, C.D., S.K. Babey, A.L. Broadfoot, R.G. Brown, L.L. Cogger, R. Gattinger, J.W. Haslett, R.A. King, D.J. McEwen, J.S. Murphree, E.H. Richardson, B.R. Sandel, K. Smith, and A.V. Jones, "An Ultraviolet Auroral Imager for the Viking Spacecraft,", Geophys. Res. Letts., 14, 4, 1987.

${ }^{15}$ Lichten, S.M. and J.A. Estefan, "High-Precision Orbit Determination for High-Earth Elliptical Orbiters Using the Global Positioning System," AIAA-90-2954-CP.

${ }^{16}$ Wu, S.C., "Differential GPS Approaches to Orbit Determination of High-Altitude Earth Satellites," AAS, $85-430,1985$.

${ }^{17}$ Monarch GPS Spaceborne User Receiver Data Brochure, Motorola Inc, Strategic Electronics Division, Chandler, AZ, 1989.

${ }^{18}$ Talley, P.E., "GPS Space Navigation and Pointing System (SNAPS)," AAS, 66, 88-034, 1988.

${ }^{19}$ "Spacecraft Applications of Advanced Global Positioning System Technology," Final Report for Contract No. NAS 9-17781, Texas Instruments Incorporated, Plano, Texas, 1988.

${ }^{20}$ Weiss, I.M., B.E. Brumbaugh and J.S. Avrin, "Attitude Determination Using GPS Measurement Techniques," AAS, 66, 88-033, 1988.

${ }^{21}$ Space Mission Analysis and Design, J.R. Wertz and W.J. Larson, Eds., Kluwer Academic Publishers, Dordrecht, Holland, 1991 .

22Private communication with Mountain Optech, Inc.

${ }^{23}$ Private communication with Odetics, Inc.

${ }^{24}$ Agrawal, B.N., "Design of Geosynchronous Spacecraft," Prentice-Hall, Inc., Englewood Cliffs, NJ, 1986. 